\title{
AN APPROACH TO THE DESIGN OF NETWORKED EMBEDDED SYSTEMS
}

\author{
Miroslav Sveda and Radimir Vrba \\ Brno University of Technology, Brno, Czech Republic \\ sveda@fit.vutbr.cz; vrbar@feec.vutbr.cz
}

\begin{abstract}
The paper presents an approach to formal specification, verification and prototyping of network applications ranging from large information systems down to small components embedded e.g. in mobile devices. Main attention focuses both on architectural and behavioral specifications of either reactive or real-time activities utilizing either structured or object-oriented approach depending on application requirements. This contribution discusses in more detail executable specifications and rapid prototyping for structured design and structural specifications and verifications for object-oriented design. The paper presents two original tools developed for that purpose: (i) Asynchronous Specification Language and (ii) Class Specification Language. Copyright $($ C 2005 IFAC
\end{abstract}

Keywords: embedded systems, design systems, formal specification, computer communication networks, sensor systems.

\section{INTRODUCTION}

Current embedded system applications are software, hardware, and communication intensive, and their functional, performance, reliability, and security requirements mandate tightly integrated information processing and physical platform behavior. Development of such complex systems necessarily stems from formal specifications and their verification and prototyping.

The paper presents particular results of a current research that deal with executable specifications and rapid prototyping for structured design and with structural specifications and verifications for objectoriented design. The presented work focuses on a class of networked systems embedded in industrial applications.

The developed methods and tools cover front-end phases of design cycles, namely formal specification and rapid prototyping both of architecture and behavior of applications under design. The approach can be explained as an employment of complex reactive systems' universal development scheme, designed by Harel (2001), for the domain of industrial distributed computer-based systems. That scheme leads from a requirements capture method to full behavioral descriptions of system parts, and from there to final implementation.

\section{STATE OF THE ART}

Requirements on current embedded system applications include both functional and nonfunctional constraints on real-time, safety and security properties (Melhart and White, 2000) that should be formally specified and verified or, at least, properly explored before they are designed in detail and implemented (Lamport, 2002). Moreover, the specification approach should either conform or suitably complement anticipated design methods, namely structured or object-oriented techniques (Wieringa, 1998). Some applications demand to distinguish at the beginning structural and behavioral specifications while later on they request to integrate those two approaches to enable a complex viewpoint to study interdependencies (Lamport, 2002).

The design of well thought-out information system applications should consider namely functionality and dependability measures (Hessami, 2004). Functionality means services delivery in the form and time fitting requirement specifications, where the service specification is an agreed description of the 
expected service. Functionality properties should be realized efficiently and cost-effectively, so reachable performance and simplicity of implementation belongs to the checked properties. Dependability is that property of a system that allows reliance to be justifiably placed on the service it delivers. A failure occurs when the delivered service deviates from the specified service. Dependability measures consist of reliability, availability, security, safety and survivability, from which this project focuses on safety, which is the ability to deliver service under given conditions with no catastrophic affects, and security, which is the ability to deliver service under given conditions for a given time without unauthorized disclosure or alteration of sensitive information. Safety attributes add requirements to detect and avoid catastrophic failures. Security attributes add requirements to detect and avoid intentional faults.

\section{DESIGNS WITH FORMAL SPECIFICATIONS}

Specification is a written or graphical description (i) of what system is supposed to do (behavioral specifications) or (ii) of system architecture (structural specifications). A formal specification asserts that a description has precise and unambiguous semantics. The language of specification has to fit purposes of specification and be appropriate for a description of the system. The presented design approach employs both structured and object-oriented specification styles through appropriate specification languages aiming not only at either structured or object-oriented developments, but also at both behavioral and architectural specifications including their rapid prototyping in frame of a design.

This section discusses tools that enable to utilize structured and object-oriented specifications of a class of computer-based systems that can be characterized as networked embedded systems in industrial applications. The developed methods and tools cover front-end phases of design cycles, namely formal specification and rapid prototyping both of architecture and behavior of applications under design.

\subsection{Formal specification tools}

Formal specification concepts employed respect both structured and object-oriented design depending on the considered target implementation approach or on the role of a tool in the development process (Wieringa, 1998). For structured behavioral specifications of reactive systems, process algebra CSP, temporal logic LTL and related transition systems in frame of the model checker SPIN (Holzmann, 1997) and the prover PVS (Owre et al., 1992) have been employed. Additionally for realtime systems, model checker UPPAAL (Kim et al., 1997) and related real-time automata have been used. In addition to the above mentioned freely available and well-known tools, the following means have been newly developed in frame of the presented research: (i) real-time executable specification language with rapid prototyping technique for structured design (Sveda and Vrba, 2001); and (ii) extension of typed object calculus FOB1 that deals with object-oriented architectural specifications (Rysavy and Sveda, 2003). The next subsections introduce main concepts of those new tools.

\subsection{Structured specifications}

The Asynchronous Specification Language (ASL) employs distributed processes with message passing. The real-time operational semantics of the language stems from the event-count model of local time, which represents a concept of physical timing stemming from some periodic physical oscillation whose frequency fits measurements of the duration of local process actions. Timing semantics can be derived from logical time, which is a partial ordering of events in the system, and from a physical generator of periodic events, which implements a real-time clock. An event-count, E, counts the number of a specific type of events that have occurred during execution. Each event occurrence invokes the implicit operation ADVANCE(E): $\mathrm{E}:=\mathrm{E}$ +1 . The explicitly callable operation $\operatorname{AWAIT}(\mathrm{E}, \mathrm{s})$ suspends the calling process until the value of $E$ is at least s. The calls AWAIT(E, s) can reset the current values of $\mathrm{E}$, enabling relative counting. An eventcount monitors either a prescribed type of asynchronous external events or periodic internal events that an internal timer circuit implements as local-time clock ticks. The following primitives relate to process specification, timing, communication, and control:

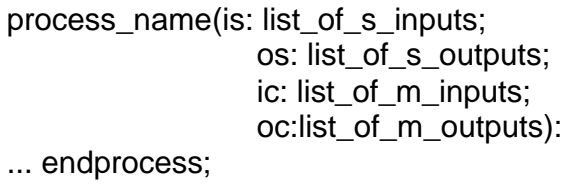

... endprocess;

wait(_, timeout);

wait(event, _);

wait(event, timeout, test);

send(message, destination);

loop ... $[\ldots \text { when }<\text { cond }>\text { action } \ldots \text { exit }]^{\star} \ldots$ endloop;

Each of asynchronous processes can be equipped by its individually timed local clock, can receive messages through input buffer, and can send messages to other, directly or indirectly addressable processes. Process header contains in parentheses lists labeled by is, os, ic, and oc that act as the interface with the process' environment. The language distinguishes between signal inputs or outputs, which denote communication events carrying either value or signaling their occurrence, and message inputs or outputs as typed asynchronous channels between couples of processes. Those signals and messages declare the interprocess synchronization and communication, whose operations are driven by the statements wait(_, 
timeout), wait(event, _), wait(event, timeout, test), and send(message, destination).

The primitive wait(_, timeout) suspends a process for the interval defined by the value timeout. Operational semantics can be obtained through the event-count abstraction introduced above: in this case, an event is every tick of the local clock, so the related operation is AWAIT(local_ticks, timeout_value). For the primitive wait(event, _), which suspends a process until the specified event (external signal or message) appears, the model operation is AWAIT(event_type, 1). The semantics of the combined statement wait(event, timeout, test) requires two event-counts: the first anticipates the specified event and the second, with a lower priority, monitors the local clock. The reason of process activation can be checked through the value of the logical variable test: when the value is true, the event occurred within the interval timeout.

The primitive send(message, destination) implements asynchronous communication with nonblocking semantics. To respect different local clocks, a special clocking that is common for the source and the destination controls the information transfer; however, the nodes communicate asynchronously by message passing through an input buffer at the destination. The input of a message induces the event for the related operation AWAIT(message,1). If any synchronization is required, it must be described explicitly using wait statements.

The control structure primitives loop ... endloop delimit an indefinite cycle, which is exited upon a true result of testing the condition following the primitive when. Consequently, the statements, which occur between the action and exit primitives and which follow the endloop primitive, are executed. This structured statement enables to extend the language with additional control structures by simple macro-like text replacements such as

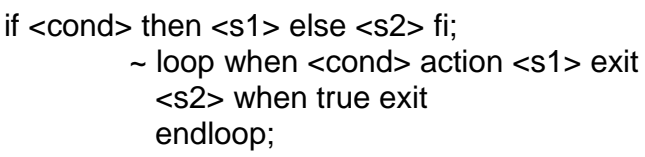

timeloop(timeinterval) ... endloop;

$$
\sim \text { loop ... wait( } \_ \text {, interval) endloop; }
$$

Actually, the control structure timeloop(timeinterval) ... endloop specifies an isochronous loop, which is periodically initiated whenever the timeinterval expires and which can be exited like the indefinite cycle. The operation AWAIT(local_ticks, timeinterval_value) defines the exact semantics of timing these initiations.

The associated rapid prototyping, which makes ASL specifications executable, arises from attribute grammar and Prolog employment. Any Prolog interpreter can drive expansion of an ASL specification into the related executable code. This expansion is based on an attribute grammar specifying both syntax and static semantics by a definite clause grammar and Prolog rules. It provides a simple language translator prototype, which tackles the ASL as the input language, and a target executable language as the output language.

The resulted prototyping technique uses interconnected node prototype boards with microprocessors equipped with a simple real-time operating system kernel. While the timing and communication primitives are mapped onto relevant real-time executive services and communication services of the operating system kernel, the rest of ASL specification is prototyped by the executable code generated with the help of the Prolog translator prototype introduced above.

\subsection{Object-oriented specifications}

The related specification language covers language constructs for description of definitions and assumptions on specification in the form of logical formulas. The specifications and assumptions provide for the proof system that verifies whether a specification is valid under the given assumptions. The specification language consists, from a structural point of view, of the language of predicate logic and the language of object calculus. Their synthesis provides a language with expressive power of higherorder logic. In terms of logic, the language contains standard predicates logical symbols, i.e. quantifiers and propositional connectives, and constants as objects defined by terms of object calculus. Since terms are interpretable in the object calculus and the language allows quantification over the set of constants, the expressive power is equal to higherorder logic. Therefore, the Gentzen deduction system may be used as a formal proof system for this language.

A specification consists of a set of classes that forms a model of the specified system. The reasoning about specification involves the use of above-mentioned sequent proof system. Because the specification language in this case is object-based, the classes are represented as special objects. A class is a basic structure of specifications that covers an implementation of objects and logical judgments on properties of objects.

The logic represents higher-order theory based on typed object calculus. It consists of a small set of primitive syntactic forms. An object is defined as a collection of attributes. The following two operations only can function on objects: (i) attribute selection a.l that results to the term obtained as an evaluation of the attribute body, and (ii) attribute update that has form a.l $\leftarrow$ b. The letters $a, b$ represent terms of the language and I is a label. Computational semantics of the calculus for both operations arise from the rules for reduction relation. A select operation provides reduction to a term that arises from the body of a selected attribute in which all occurrences of the bind variable are replaced by the object supplying the selected attribute. The result of update operation defined by reduction relation provides a new object 
identical with the target object up to the update attribute, which body is that of the updating term.

The logic includes a type theory constraining the set of well-formed terms. Typing rules of the calculus permit subtyping and provide special treatment with bool type. Although the language does not contain functions, they can be easily inferred as simple objects. A function abstraction $\lambda(x: A)$.a of type $A \rightarrow$ $B$ denotes the structure $[x=\zeta(s: T) s . x$, val $=\zeta(\mathrm{s}: T)$ a $\{x \leftarrow s\}]$ provided that $T \equiv[x: A$, val : $B]$. Then a function application $\mathrm{MN}$ is directly given as (M: $\mathrm{x} \leftarrow$ $\mathrm{N})$.val. Instances of bool type represent conditional expressions from the computational viewpoint and serve as constants of propositional types considering theirs logical meaning. The propositional connectives are introduced as a set of constants with usual meanings. Moreover, quantifiers and predicates are introduced inside the object language. To model classes, predicates allow writing constraints on types that delimit sets of objects satisfying intended conditions. Subtype creation uses operator + for denoting that a new type is obtained by adding new attributes to the old type.

The logic calculus of objects provides a suitable formal environment for specifying and logical reasoning with properties of objects. However, writing specifications directly in this calculus is tedious. More practical notation, the Class Specification Language (CSL), enables to write compact specifications, but preserves possibility to transform any specification straightforwardly to the object calculus whenever required for reasoning.

A class is defined by specifying all of its visible properties. The term property means in this case a field that represents the state of an object, or an observer that serves for the read-only access to an object, or a modifier whose execution can change the state of an object. A field declaration includes the field name and the field class. Specification of a field may be refined using invariant statement.

Field fieldName : fieldClass

Inv fieldInv = formula

Modifier and observer methods include definitions consisting of method's name, arguments, and a pair of constraints. Declarations differ for modifiers that disable user to specify a result of the method. Due to modifiers, declarations always evaluate to the object reflecting performed changes. Constraints may involve variables referencing to actual objects and variables denoting specified arguments of the method.

observer methodName(, arg : argClass, ...) : retClass pre methodPre $=$ formulaPre

post methodPost $=$ formulaPost

The language CSL enables to define a new class by application of the single inheritance. An inherited class automatically receives all fields and methods of its parent class. To handle inheritance properly, a schema for definition of invariants and conditions of inherited fields and methods is needed. Considering that class B inherits class A, then the specification of classes has to preserve inheritance constraints assuming each inherited field and method in the schema as following:

$$
\begin{aligned}
& \text { fieldInv }{ }^{B} \Rightarrow \text { fieldInv }^{A} \\
& \text { methodPre }{ }^{A} \Rightarrow \\
& \text { methodPre }^{B} \wedge \text { methodPost }^{B} \Rightarrow \text { methodPost }^{A}
\end{aligned}
$$

Defining inheritance constraints in this manner enables method overriding. The precondition of the overridden method relaxes constraints of method execution, contrary to post-conditions that involve additional constraints.

The logic calculus defines directly certain common classes as they depend on particular aspects of the calculus. The class of booleans is defined simply as a predicate on propositional type. The logic evaluates all possible instances of this class to $\mathrm{T}$ and $\mathrm{F}$ objects declared previously as abbreviations. The class of natural numbers exploits recursive object type. It consists of three attributes; two of them serve as links to predecessor and successor objects. The iszero attribute marks the numeral zero. Usual notations 0 , 1,2 ... explicitly denote related numerals.

\section{CASE STUDY}

This section demonstrates the above-introduced concepts and tools applied to development of a gaspipes pressure analyzer consisting of pressure sensors interconnected by Internet. The application is based on the IEEE 1451 family of standards, which is introduced in subsection 4.1. Subsection 4.2 explains a subset of the application functions selected for formal specifications in the rest of this section. To provide examples of specification styles using developed tools, the next two subsections present selected facets of the pressure analyzer specification. While subsection 4.3 demonstrates structured specifications using ASL, subsection 4.4 exemplifies object-oriented specifications using the developed class specification language.

\subsection{IEEE 1451.1 architecture}

The IEEE 1451 consists of the family of standards for a networked smart transducer interface that include namely (i) a smart transducer software architecture, 1451.1 (IEEE 1451.1 Standard for a Smart Transducer Interface for Sensors and Actuators -- Network Capable Application Processor Information Model, IEEE, New York, April 2000), targeting software-based, network independent, transducer applications, and (ii) a standard digital interface and communication protocol, 1451.2, for accessing the transducer or a group of transducers via a microprocessor modeled by the 1451.1. The next three standards extend the original hard-wired parallel interface 1451.2 to serial multidrop 1451.3, mixed-mode (i.e. both digital and analog) 1451.4, and wireless 1451.5 interfaces. 
The 1451.1 software architecture provides three models of the transducer device environment: (i) an object model of a network capable application processor (NCAP), which is the object-oriented embodiment of a smart networked device; (ii) a data model, which specifies information encoding rules for transmitting information across both local and remote object interfaces; and (iii) network communication model, which supports client/server and publishers/subscribers paradigms for communicating information among NCAPs. The standard defines a network and transducer hardware neutral environment in which a concrete sensor/actuator application can be developed.

The object model definition encompasses a set of object classes, attributes, methods, and behaviors that specify a transducer and a network environment to which it may connect. This model uses block and base classes offering patterns for one Physical Block, one or more Transducer Blocks, Function Blocks, and Network Blocks. Each block class may include specific base classes from the model. The base classes include Parameters, Actions, Events, and Files, and provide component classes.

All classes in the model have an abstract or root class from which they are derived. This abstract class includes several attributes and methods that are common to all classes in the model and provide a definition facility for instantiation and deletion of concrete classes including attributes. Block classes form the major blocks of functionality that can be plugged into an abstract card-cage to create various types of devices. One Physical Block is mandatory as it defines the card-cage and abstracts the hardware and software resources that are used by the device. All other block and base classes can be referenced from the Physical Block.

The Transducer Block abstracts all the capabilities of each transducer that is physically connected to the NCAP I/O system. During the device configuration phase, the description is read from the hardware device what kind of sensors and actuators are connected to the system. The Transducer Block includes an $\mathrm{I} / \mathrm{O}$ device driver style interface for communication with the hardware. The I/O interface includes methods for reading and writing to the transducer from the application-based Function Block using a standardized interface. The I/O device driver provides both plug-and-play capability and hot-swap feature for transducers.

The Function Block provides a skeletal area in which to place application-specific code. The interface does not specify any restrictions on how an application is developed. In addition to a State variable that all block classes maintain, the Function Block contains several lists of parameters that are typically used to access network-visible data or to make internal data available remotely. The Network Block abstracts all access to a network employing network-neutral, object-based programming interface supporting both client-server and publisher-subscriber paradigms for configuration and data distribution.

\subsection{Pressure analyzer}

In the transducer's 1451.1 object model, basic Network Block functions initialize and cover communication between a client and the transducer. The client-server communication style, which in this application covers configurations of transducers, is provided by two basic Network Block functions: execute and perform. The standard defines a unique ID for every function and data item of each class.

If the client wants to call any of the functions on server side, it uses command execute with the following parameters: ID of requested function, enumerated arguments, and requested variables. On server side, this request is decoded and used by the function perform. That function evaluates the requested function with the given arguments and, in addition, it returns the resulting values to the client. Those data are delivered by requested variables in execute arguments.

\subsection{Structured specifications by ASL}

The following example demonstrates the ASL specification of a client accessing the transducer. This specification includes in form of comments the most important references to sections of the IEEE 1451.1 standard.

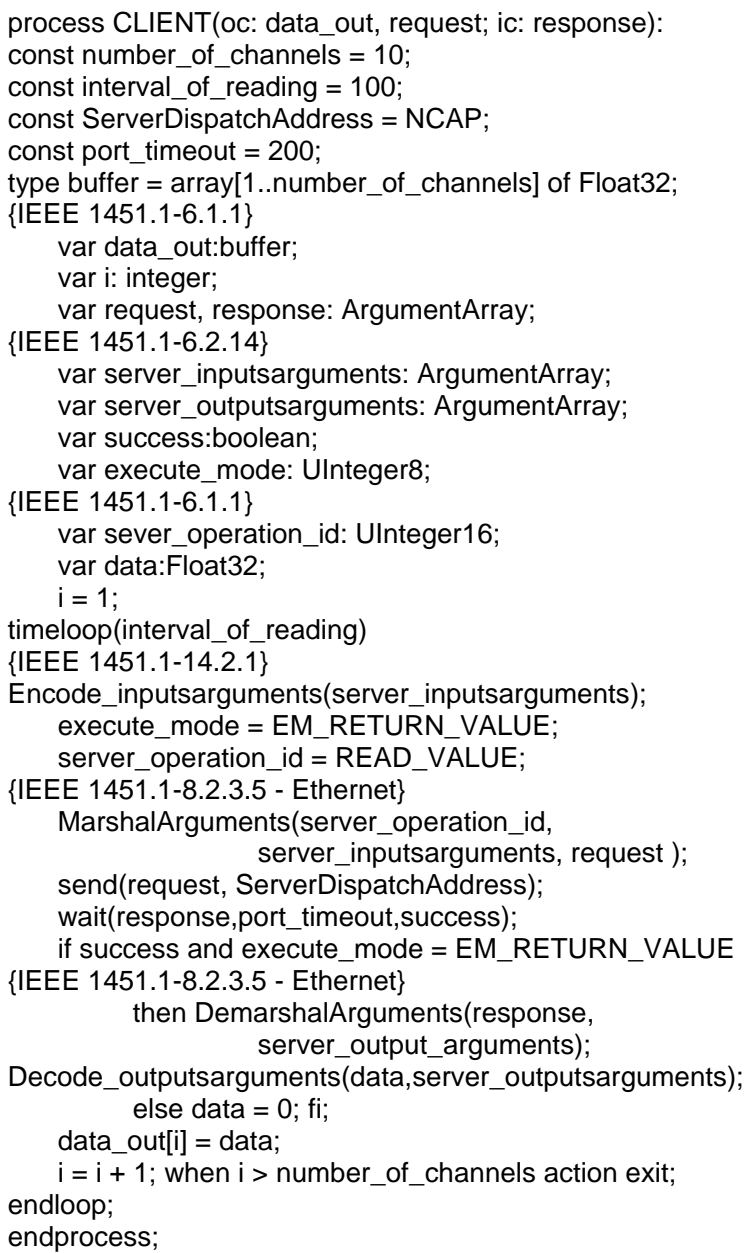




\subsection{Object-oriented specifications by CSL}

The following example demonstrates the class language specification of the NCAP Block class in frame of the class hierarchy of Block Objects. The Block abstract class provides the root for the class hierarchy of all Block Objects. The BlockMajorState type is enumeration of possible block states. The state blUninitalize is reserved for local activities related to creating that Block Object and performing any related local preparations. The object in the state bllnactive is able to configure its network communication properties, initialize itself and its owned object, and diagnose and maintain the block object. The working state blActive is achieved after all initialization and start-up procedures and represents the state in which object remains for the time of its normal activity.

BlockMajorState :: $\{$ blActive, bllnactive, blUninitialized IEEE1451Block :: [GetBlockMajorState : BlockMajorState, GoActive : IEEE1451Block, Golnactive : IEEE1451Block, Reset : IEEE1451Block, Initialize : IEEE1451Block, owns : IEEE1451Entity $\rightarrow$ bool] + IEEE1451Entity

A behavior of the object may implicitly change the state of this object often as a reaction on the environment stimulus. Moreover, a set of defined operations forces the object to change its state explicitly. The meaning of those operations is defined by a set of constraints. The behavior of this object constrained by meaning of these operations is specified as IEEE1451BlockBehavior invariant.

INV IEEE1451BlockBehavior(x : IEEE1451Block) $\equiv$ x.GetBlockMajorState $\leftrightarrow$ bllnactive

$\supset$ x.GoActive.GetBlockMajorState $\leftrightarrow$ blActive

$\wedge$ x.GetBlockMajorState $\leftrightarrow$ blActive

$\supset$ x.Golnactive.GetBlockMajorState $\leftrightarrow$ bllnactive

$\wedge$ x.Reset.GetBlockMajorState $\leftrightarrow$ blUninitialized

$\wedge \neg$ x.GetBlockMajorState $\leftrightarrow$ blActive

$\supset$ x.Initialize.GetBlockMajorState $\leftrightarrow$ bllnactive

The NCAP Block class provides resources and operations within an NCAP process to support Block, Service, and Component management.

NCAPBlockState :: \{nbllnitialized, blUninitialized, nbErro\} IEEE14 51Block :: [GetNCAPBlockState : NCAPBlockState, RegisterObject : IEEE1451Block, DeregisterObject: IEEE1451Block,

registers : IEEE1451Entity $\rightarrow$ bool] + IEEE1451Block

The state space derived from Block object is divided into the more specialized substates that reflect purpose of NCAP Block objects. The state of object, which is stored in GetNCAPBlockState item, may obtain values of NCAPBlockState type.

\section{CONCLUSIONS}

The presented project deals with front-end parts of networked, distributed system application designs. The project targets creation of a formal specification, verification and prototyping framework for network applications ranging from large information systems down to small components embedded e.g. in mobile devices. Main attention focuses both on architectural and behavioral specifications of either reactive or real-time activities utilizing either structured or object-oriented approach depending on application requirements. Formal specification tools considered include temporal logics, real-time logics, process algebras and transition systems. The implementation and integration phases of the project provide pilot versions of techniques and tools for conceptual design, for architectural specifications, for reactive and real-time system behavior specifications, and for rapid prototyping. A case study respecting real world constraints demonstrates utilization of the developed approach.

\section{ACKNOWLEDGEMENT}

The research has been partly supported by the Grant Agency of the Czech Republic through the grant GACR 102/05/0723: A Framework for Formal Specifications and Prototyping of Information System's Network Applications, and through the grant GACR 102/05/0467: Architectures of Embedded Systems Networks.

\section{REFERENCES}

Harel, D. (2001) From Play-In Scenarios to Code: An Achievable Dream. IEEE Computer, 34(1), 53-60.

Hessami, A.G. (2004) A Systems Framework for Safety and Security: The Holistic Paradigm. Systems Engineering, 7(2), 99-112.

Holzmann, G.J. (1997) The Model Checker Spin. IEEE Transactions on Software Engineering, 23(5), 279-295.

Kim, G., L. Pettersson, P. Pettersson and Wang, Y. (1997) Uppaal in a Nutshell. Int. Journal on Software Tools for Technology Transfer, 1(1-2), 134-152.

Lamport, L. (2002) Specifying Systems, AddisonWesley, Boston, USA.

Melhart, B. and S. White (2000) Issues in Defining, Analyzing, Refining, and Specifying System Dependability Requirements. Proceedings of the IEEE Conference and Workshop ECBS'2000, IEEE Computer Society Press, Edinburgh, Scotland, 334-340.

Owre, J.M., J.M. Rushby and N. Shankar (1992) PVS: A Prototype Verification System. In: Automated Deduction, (D. Kapur, Ed.), Lecture Notes in Artificial Intelligence, Vol.607, pp.748-752, Springer, New York, USA.

Rysavy, O. and M. Sveda (2003) A Minimal Formal Language for Object-Oriented Specifications. Proceedings of the IEEE TC-ECBS and IFIP WG10.1 Joint Workshop on Formal Specifications of Computer-Based Systems. University of Stirling, UK, 35-40.

Sveda, M. and R. Vrba (2001) Executable Specifications for Distributed Embedded Systems. IEEE Computer, 34(1), 138-140.

Wieringa, R. (1998) A Survey of Structured and Object-Oriented Software Specification Methods and Techniques. ACM Computing Surveys, 30(4), 459-527. 